\title{
Congenital short pancreas: a report of two cases
}

\author{
N H GILINSKY, G DEL FAVERO, P B COTTON, AND W R LEES \\ From the Departments of Gastroenterology and Radiology, The Middlesex Hospital, London
}

SUMmARY We report two adult cases with partial agenesis of the pancreas. Their radiological features, including endoscopic retrograde pancreatography are demonstrated. The significance of developmental anomalies involving the pancreas is emphasised.

Congenital abnormalities of the pancreatic duct system, long recognised by anatomists, ${ }^{1-3}$ have assumed a more widespread importance since the introduction of endoscopic retrograde pancreatography (ERP). The pancreas is formed by the fusion of dorsal and ventral buds. This is followed by the formation of ductal, secretory, and endocrine systems. The more frequent anomalies encountered are those of malfusion, migration, and duplication. ${ }^{45}$ Agenesis of a pancreatic bud, either partial or total, is an exceptionally rare finding in adults. $^{6-9}$ To our knowledge, only seven examples have been reported, and only one case involving a living adult exists in the English literature. ${ }^{7}$ We wish to report two additional cases.

\section{Case 1}

A 40 year old housewife was referred in 1976 after a one year history of episodic right upper quadrant pain. Cholecystography showed gall stones which were confirmed at cholecystectomy. In 1978 she required radioiodine for thyrotoxicosis. Two years later she was seen for depression and upper abdominal discomfort. There was no background of alcoholism, drug ingestion, or abdominal trauma, and no family history of note. She was clinically and biochemically hypothyroid. Liver function tests, serum lipids and amylase were normal. Ultrasonography showed normal bile ducts and a normal pancreatic head; however, the neck was thin and tapered to nothing. No pancreatic tissue could be identified in the region of the body or tail. An ERP showed an abnormal duct system originally interpreted as being consistent with a pancreas divisum. The abdominal discomfort settled with

Address for correspondence: Dr P B Cotton, Department of Gastroenterology, The Middlesex Hospital. Mortimer Street. London WIN 8AA. Received for publication 18 May 1984 treatment of the hypothyroidism. The ducts of Santorini and Wirsung were both shown to be present at repeat ERP in 1981 (Figs. 1 and 2). A ductal system could not be shown in the body or tail. No evidence of pancreatitis was present. A revised diagnosis of a 'congenitally short pancreas' was made. She was asymptomatic when reassessed in 1983. Her biochemistry was normal as were tests of pancreatic function (Table). Ultrasonography (Aloka SSD 250) revealed no change from 1980. An isotope scan (selenium-methionine) could not identify any definite pancreatic uptake. Computed tomography (IGE 8600) showed a normal head of pancreas; no body or tail could be found (Fig. 3).

\section{Case 2}

A 36 year old cleaner was referred in 1982 for assessment of abdominal pain. He was well until 1978 when he developed insulin dependent diabetes.

Table Biochemistry and pancreatic function testing

\begin{tabular}{llcc}
\hline & $\begin{array}{l}\text { Normal } \\
\text { value }\end{array}$ & Case I & Case 2 \\
& 15 & 12 & 8 \\
\hline Serum bilirubin $(\mu \mathrm{mol} / \mathrm{l})$ & $36-50$ & 40 & 45 \\
Serum albumin $(\mathrm{g} / \mathrm{l})$ & $2 \cdot 20-2 \cdot 55$ & $2 \cdot 42$ & $2 \cdot 48$ \\
Serum calcium $(\mathrm{mmol} / \mathrm{l})$ & & & \\
Serum alkaline & $100-280$ & 123 & 166 \\
$\quad$ phosphatase (IU/l) & $<40$ & 15 & 38 \\
Serum aspartate & $<300$ & 207 & 256 \\
$\quad$ aminotransferase (IU/l) & $<200$ & 105 & 183 \\
Serum amylase (IU/l) & $<19$ & 8 & 22 \\
Faecal weight (g/day) & $>50$ & 67 & 50 \\
Faecal fat (mmol/day) & $\dagger$ & Normal & $\ddagger$ \\
PABA excretion* (\%) & & \multicolumn{2}{c}{} \\
Glucose tolerance test & & &
\end{tabular}

* Standard 0.5 g N-benzoyl-L-tyrosyl-p-aminobenzoic acid test.

+ Standard 75 g test.

$\ddagger$ Known diabetic, therefore not indicated. 


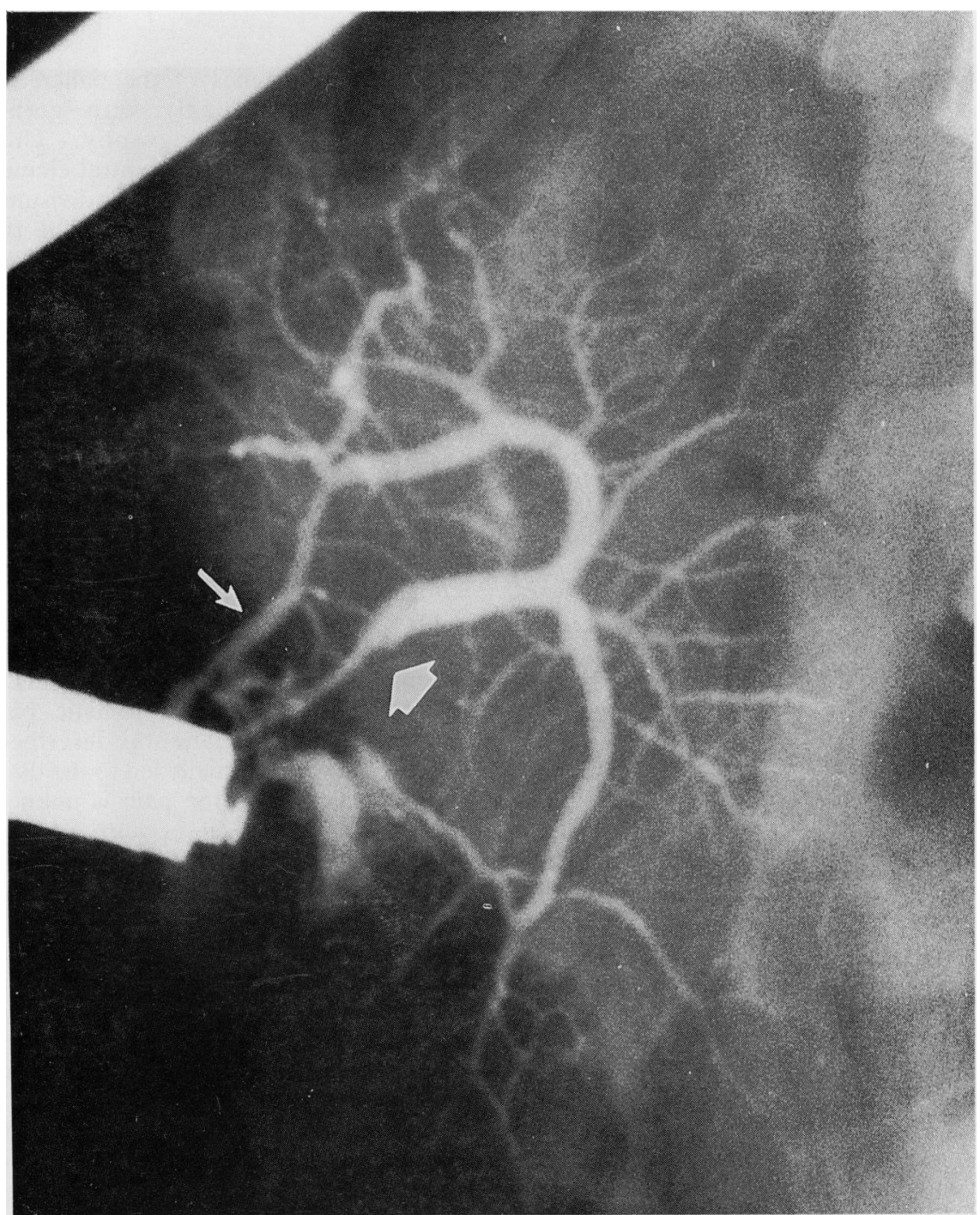

Fig. 1 Case 1. Pancreatogram showing an abnormally short duct system. The main papilla has been cannulated outlining Wirsung's duct (lower arrow). Contrast has flowed retrogradely into Santorini's duct to the accessory papilla (upper arrow) and has leaked out into the duodenum. The ducts themselves are normal.

Raised liver enzymes were found repeatedly in 1981, and a percutaneous liver biopsy was done. This resulted in severe abdominal pain necessitating laparotomy at which a perforated gall bladder was removed. The liver histology (percutaneous and operative) was normal. The operation was followed by persistent epigastric discomfort, aggravated by meals and anxiety. There was no loss of weight, change in bowel habit, relief from a low fat diet, or cimetidine. He had consumed four pints of beer daily for about six years until 1981. There was no background of abdominal trauma, or previous abdominal pain and no relevant family history. In 1982 an abdominal radiograph revealed calcification in the head of the pancreas. Ultrasonography showed an abnormal echogenic pattern in the head of the pancreas, but no body or tail could be identified. An ERP revealed an abnormal duct configuration thought to be consistent with a ventral pancreas. A repeat ERP a few months later, 


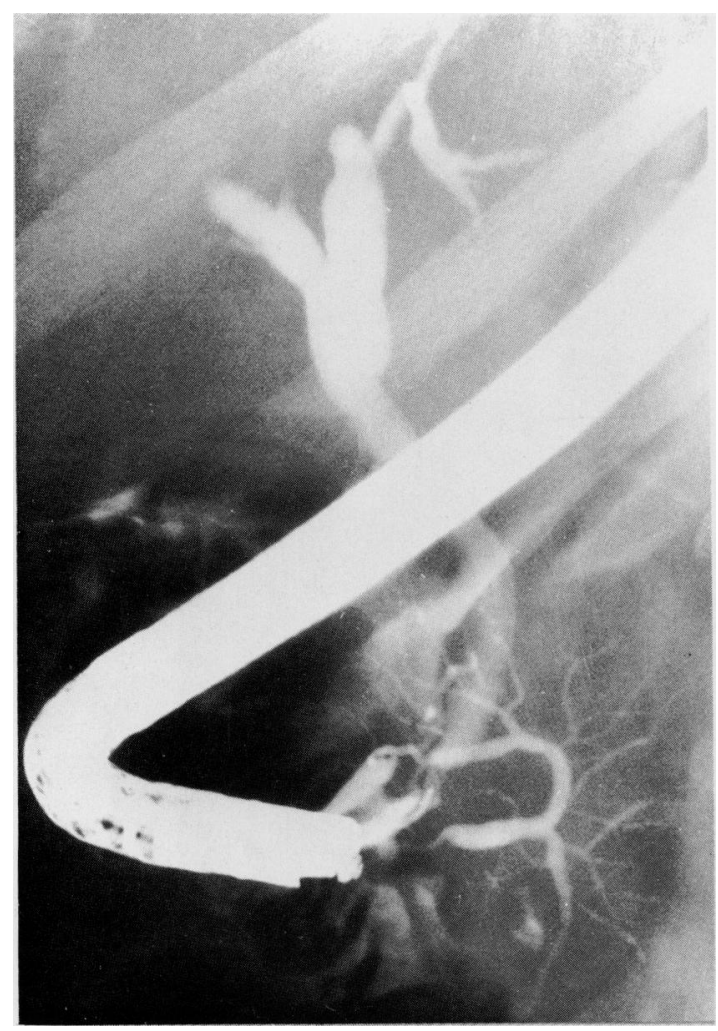

Fig. 2 As for Fig. 1 except that the bile duct is now outlined and more contrast is flowing out into the duodenum via the accessory papilla.

however, showed that the accessory papilla was distal to the main one. No other papilla could be found. The ducts of Santorini and Wirsung were identified. Injection of contrast into the main papilla resulted in retrograde spillage into the duodenum via the accessory papilla (Figs. 4 and 5). No contrast entered the body of the gland, and there were no signs suggesting pathological obstruction; there were only minor calibre variations in the ducts themselves, despite the presence of calcification. The results of biochemistry and pancreatic function testing when reassessed in 1983 are shown (Table). An isotope scan failed to detect any definite pancreatic structure. Ultrasonography again could not identify any pancreatic tissue in the region of the body or tail. Computed tomography scanning revealed a slightly abnormal head of pancreas (Fig. 6 ); no body or tail could be identified. A revised diagnosis of a 'short pancreas', probably because of a congenital abnormality, was made.

\section{Discussion}

Congenital anomalies of the pancreas have been recognised increasingly with greater use of endoscopic pancreatography. The pancreas develops from dorsal and ventral elements. Failure of fusion may result in 'pancreas divisum', present in at least $5 \%$ of the population. ${ }^{4}$ The rarer annular pancreas occurs when the two parts fuse through a circumferential branch. Complete agenesis of the pancreas has been reported in still born fetuses, in association with multiple anomalies. ${ }^{10}$ The two patients described in this report represent examples of a 'congenital short pancreas', which must be because of partial agenesis. Both patients had a duct system which communicated with both minor and major papilla, indicating that the embryological ventral and dorsal elements had fused. We have found only seven possible examples previously reported in living adults. ${ }^{7-9}$

The abnormality in our first patient was discovered incidentally during the course of investigations for possible common bile duct stones, and represents the first asymptomatic patient to be reported. In the patients described to date, abdominal pain eventually led to the diagnosis being made. The cause of the pain is unknown and has been presumed to be because of an autonomic neuropathy in those with diabetes. ${ }^{7}$ Although no histological evidence of pancreatitis was found in open biopsy specimens, ${ }^{79}$ it is interesting to speculate whether the congenital abnormality may in fact predispose to recurrent bouts of pancreatitis and abdominal pain - as has been suggested in patients with pancreas divisum. ${ }^{4}$ Our second patient appears to have been unusually sensitive to only moderate amounts of alcohol.

Diabetes has been present in five of the seven reported cases; in some instances it substantially preceded the onset of abdominal discomfort and anatomical diagnosis. ${ }^{79}$ Secretin-pancreazymin testing was abnormal in only one of five cases where this investigation was done. ${ }^{9}$ Pancreatic exocrine dysfuction is probably less common as approximately $90 \%$ of the secretory capacity of the pancreas must be lost before significant steatorrhoea is observed. ${ }^{11}$ Simple function testing of our asymptomatic patient was normal, while the diabetic had a borderline result.

Proof of the diagnosis has been made by laparotomy in the other reports. ${ }^{7-9}$ The pancreas was not specifically examined at the operations, however, which had been performed previously in our patients. Nowadays the diagnosis can be made by careful scanning and pancreatography. The anomaly must be differentiated from pancreatic 


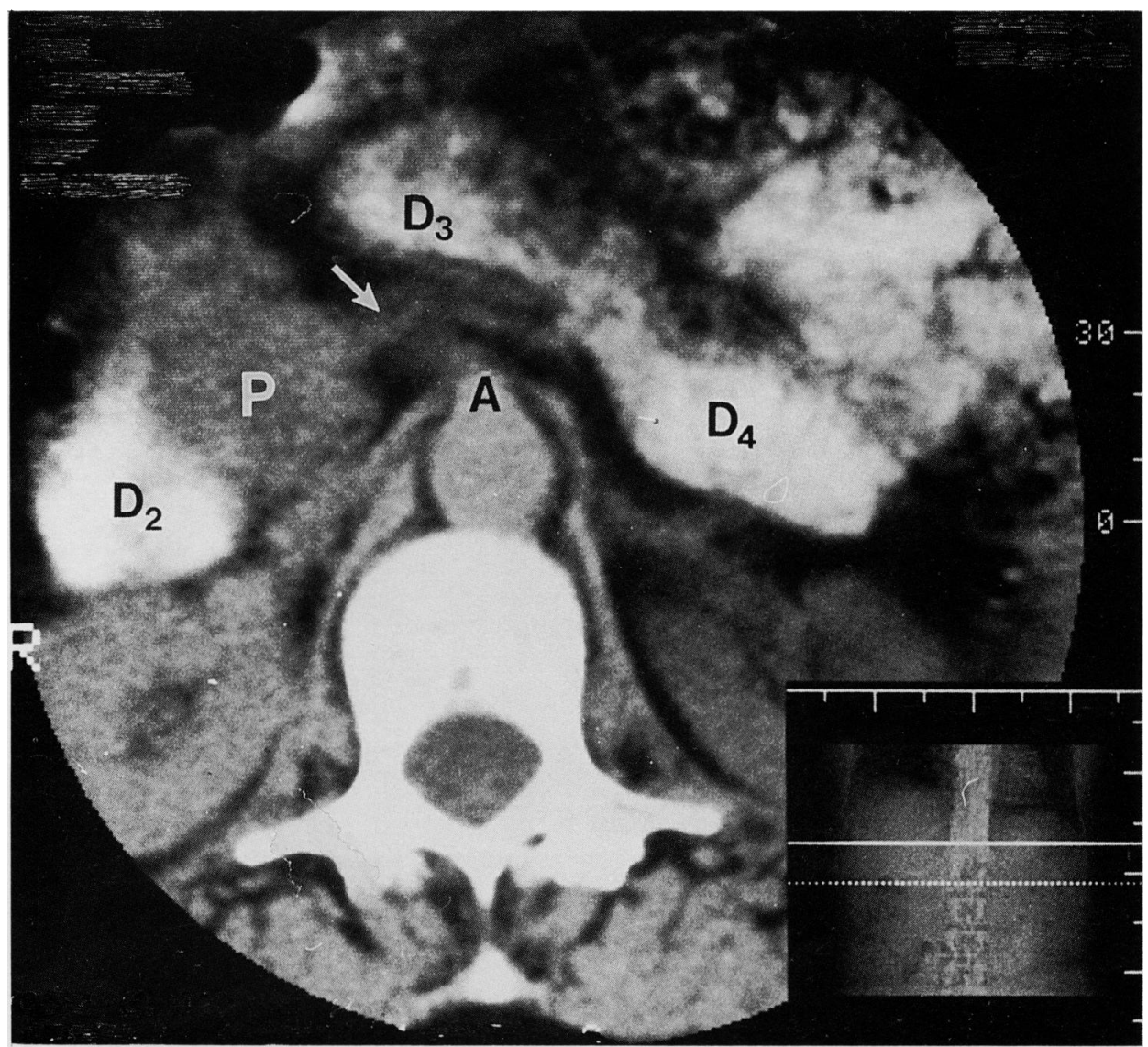

Fig. 3 Computer tomography scan (Case 1) at level of origin of the superior mesenteric artery $(A)$ showing the head of the pancreas $(P)$ only. There is contrast in the 2 nd part of the duodenum $\left(D_{2}\right)$. The $3 r d$ and 4 th parts $\left(D_{3}, D_{4}\right)$, and splenic vein (arrow) are shown.

atrophy, secondary to pancreatitis upstream of an obstructed duct. The distinction may be subtle, especially in a patient with 'partial pancreas divisum', with obstruction of the main duct at the point of the small communication between the dorsal and ventral elements (Fig.7).

The ERP features of our patients have not been documented previously. Phillip et al ${ }^{12}$ describe a patient with a ductal system limited to the head which they attributed to a 'primitive form' of annular pancreas. The first patient described by Morita et al showed a short branching main duct on radiographic criteria also consistent with a ventral pancreas. ${ }^{9}$ The computed tomography scan apparently showed atrophy of the body and tail, with a cyst in the head. Angiography was virtually normal, with only a suspicion of reduced vascularity to the tail. A mass in the tail of the pancreas was suspected in their second case when ERP showed a short and dilated main duct.

None of the previous reports mention attempts to find or exclude the existence of an accessory papilla or Santorini vestige. This should be done before ascribing a case of possible pancreatic atresia to total agenesis of the dorsal pancreas. We were able to identify main and accessory papillae in both of our patients and thus confirm that the ducts of Santorini and Wirsung (although shortened) were present. As fusion of the two pancreatic elements takes place during the second month of fetal development, we can assume in our cases that failure of the dorsal segment to develop occurred after this time. If, by definition, the main pancreatic duct opens together with the bile duct into the major papilla, then our 


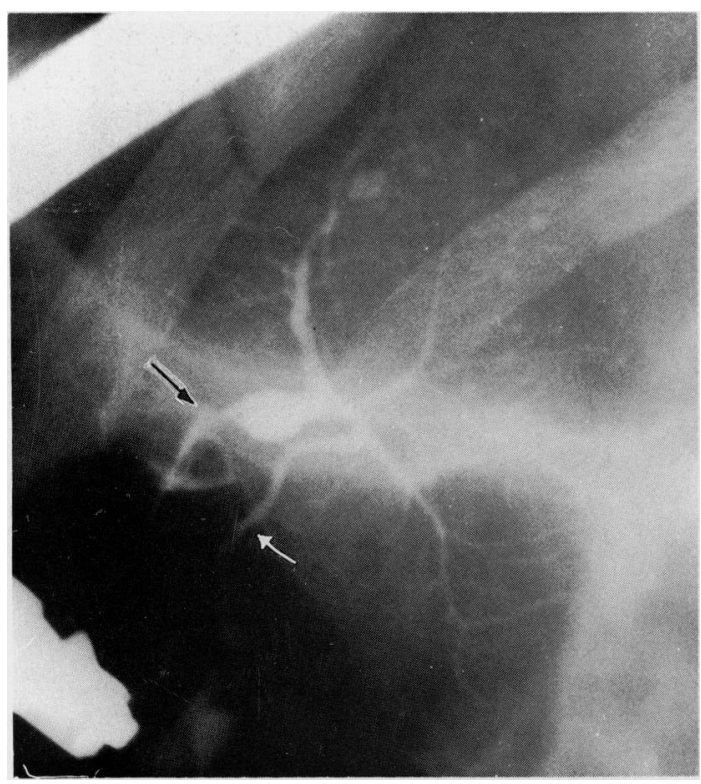

Fig. 4 Case 2. The main papilla (black arrow) has been cannulated and a very short duct of Wirsung is seen. Contrast has flowed retrogradely and has outlined a distally situated duct of Santorini (white arrow). Contrast has leaked back into the duodenum via the accessory papilla. Pancreatic calcification is present. second case shows another varient - that of a distal accessory papilla. It is possible that during the stage of rotation, the dorsal segment came to lie proximal and not distal to the ventral pancreatic element.

The anomaly described in this report should not be confused with the entity of pancreatic hypoplasia - owing to lack of differentiation in the terminal duct system. This causes hypoplasia of the exocrine tissue and thus deficient secretion. The main ducts and endocrine function are normal. The epithelial elements are replaced by fatty tissue and the organ is normal in size and shape. ${ }^{13}$

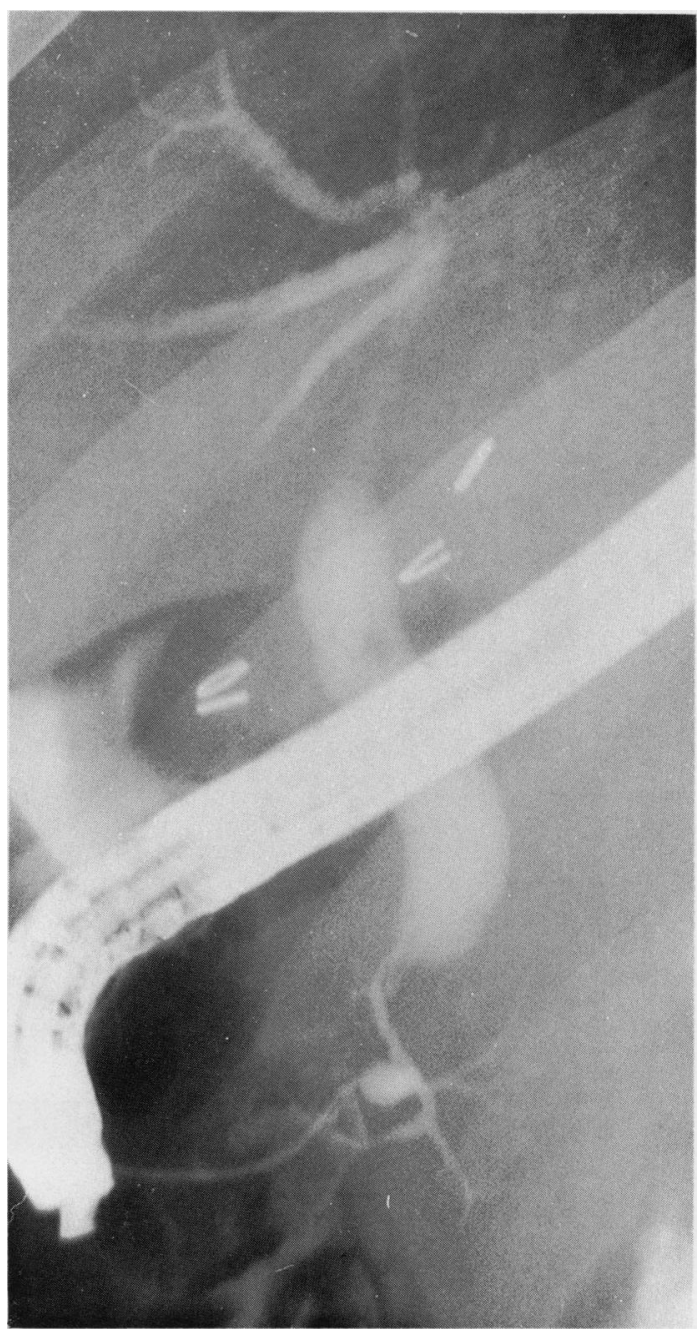

Fig. 5 As for Fig. 4 except that now the bile duct is opacified. More contrast is present in the duodenum. An air bubble is seen in the bile duct

Failure to recognise congenital anomalies of the pancreas may lead to inappropriate diagnoses being made. Several different imaging techniques may have to be used to avoid misinterpretation.

We are grateful to our many colleagues at The Middlesex Hospital for contributing in various ways to the investigation and management of these patients; and to Drs C Bartlett and R Wilkins (Radiology Department, Northwick Park Hospital), who performed the computed tomography scans. 


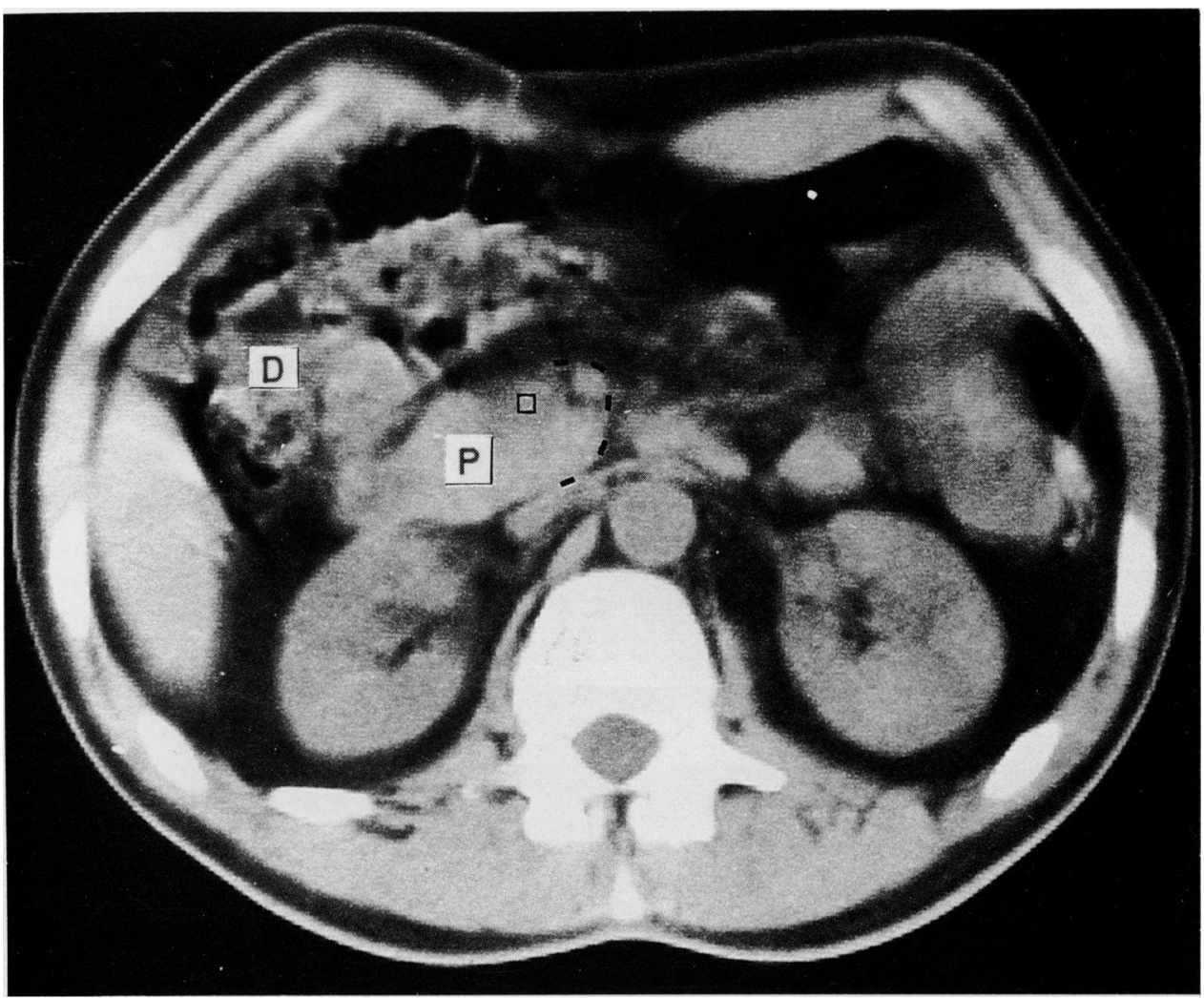

Fig. 6 Computer tomography scan (case 2) $2 \mathrm{~cm}$ below origin of superior mesenteric artery showing head of pancreas $(P)$ containing an area of slightly reduced attenuation (open square). The medial border of the gland is outlined (broken line) and duodenum (D) shown. Contrast was not well tolerated.

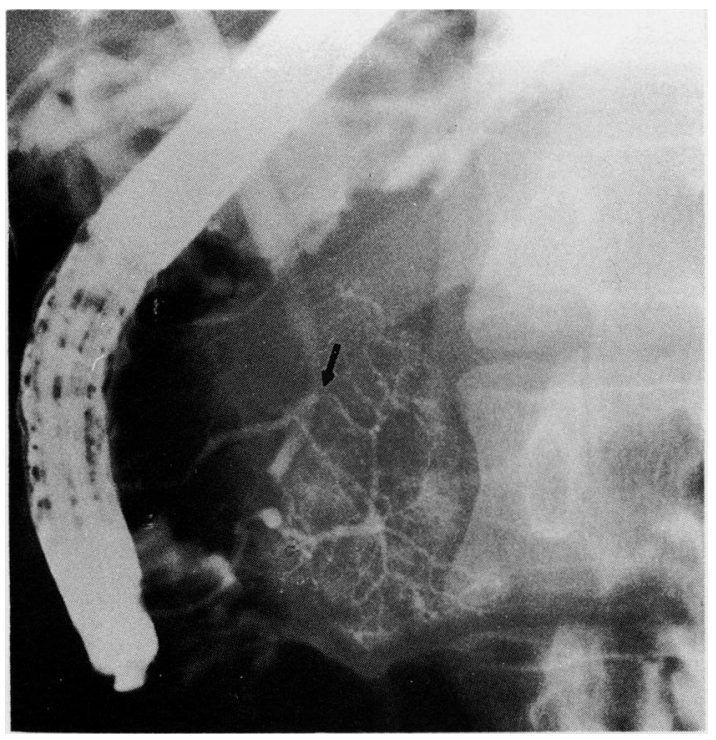

Fig. 7 Initially thought to be compatible with a congenital short pancreas, this pancreatogram shows an obstruction of the dorsal duct at the point of communication between the two segments of a partial pancreas divisum (arrow). Other imaging techniques confirmed a pseudocyst in a virtually normal sized gland. 


\section{References}

1 Bremer JL. Pancreatic ducts and pancreatic bladders. Am J Anat 1923; 31: 289-318.

2 Dawson W, Langman J. An anatomical-radiological study on the pancreatic duct pattern in man. Anat Rec 1961; 139: 59-68.

3 Belber JP, Kazuko B. Fusion anomalies of the pancreatic ductal system: differentiation from pathological states. Radiology 1977; 123: 637-42.

4 Cotton PB. Congenital anomaly of pancreas divisum as cause of obstructive pain and pancreatitis. Gut 1980; 21: $105-14$.

5 Yatto RP. Siegel JH. Varient pancreatography. Am J Gastroenterol 1983; 78: 115-8.

6 Kriss B. Zur kenntnis der hypoplasie des pankreas. Virchows Arch [Pathol Anat] 1926; 263: 591-8.

7 Lechner GW, Read RC. Agenesis of the dorsal pancreas in an adult diabetic presenting with duodenal ileus. Ann Surg 1966; 163: 311-4.

8 Guntz M, Albaret P, Joubaud F, Francois H. Absence du corps et de la queue du pancreas et pancreatite chronique calcifiante. Pancreatectomie totale. Sem Hôp Paris 1976; 52: 1863-7.

9 Morita M, Otsubo C, Kozu T et al. Aplasia of the body and tail of the pancreas - a report of two cases. Nippon Shokakibyo Gakkai Zasshi 1980; 77: 102-6.

10 Warkany J. Congenital malformations. Chicago: Year Book Medical Publishers, 1971: 729-31.

11 DiMagno EP, Go VLW, Summerskill WHJ. Relations between pancreatic enzyme outputs and malabsorption in severe pancreatic insufficiency. $N$ Engl J Med 1973; 288: 813-5.

12 Phillip J, Koch H, Classen M. Variations and anomalies of the papilla of Vater, the pancreas, and the biliary duct system. Endoscopy 1974; 6: 70-7.

13 McLean JM. Embryology of the pancreas. In: Howat HT, Sarles H, eds. The exocrine pancreas. London: WB Saunders, 1979: 1-14. 